\title{
Stationary property of the thermodynamic potential of the Hubbard model in strong coupling diagrammatic approach for superconducting state
}

\author{
V.A. Moskalenko ${ }^{1,2}$, L.A. Dohotaru ${ }^{3}$, D.F. Digor ${ }^{1}$, and I.D. Cebotari ${ }^{1}$ \\ ${ }^{1}$ Institute of Applied Physics, Moldova Academy of Sciences, Chisinau 2028, Moldova \\ ${ }^{2}$ The Bogoliubov Laboratory of Theoretical Physics, Joint Institute for Nuclear Research, Dubna 141980, Russia \\ ${ }^{3}$ Technical University, Chisinau 2004, Moldova \\ E-mail: moskalen@thsun1.jinr.ru
}

Received February 13, 2012, revised April 17, 2012

\begin{abstract}
Diagrammatic analysis for normal state of Hubbard model proposed in our previous paper is generalized and used to investigate superconducting state of this model. We use the notion of charge quantum number to describe the irreducible Green's function of the superconducting state. As in the previous paper we introduce the notion of tunneling Green's function and of its mass operator. This last quantity turns out to be equal to correlation function of the system. We proved the existence of exact relation between renormalized one-particle propagator and thermodynamic potential which includes integration over auxiliary interaction constant. The notion of skeleton diagrams of propagator and vacuum kinds were introduced. These diagrams are constructed from irreducible Green's functions and tunneling lines. Identity of this functional to the thermodynamic potential has been proved and the stationarity with respect to variation of the mass operator has been demonstrated.
\end{abstract}

PACS: 71.27.+a Strongly correlated electron systems; heavy fermions;

71.10.Fd Lattice fermion models.

Keywords: strong correlated electron system, Dyson equation, Green's function, periodic Anderson model.

\section{Introduction}

The present paper generalizes our previous work [1] on diagrammatic analysis of the normal state of the Hubbard model [2-4] to the superconducting state.

Now we shall assume the existence of pairing of charge carriers and non-zero Bogolyubov quasi-averages [5] and, consequently, of the Gor'kov anomalous Green's functions [6].

The central idea of standard BCS theory of conventional superconductivity is formation of Cooper pairs due to the presence of attractive interaction between electrons. Such attractive interaction can be of electron-phonon kind with mechanism based on the polarizability of ionic lattice in metal. After the discovery in 1986 of high-temperature superconductivity in cuprate compounds with layered perovskite structure begins the era of unconventional superconductivity with possible alternative mechanisms of superconductivity. One of such possible mechanism is spin fluctuation exchange [7] one based on the conception of spin polarization of electrons.
One of the most frequently used model for unconventional superconductivity is the Hubbard model. We shall discuss below its properties.

The main property of the Hubbard model consists in the existence of strong electron correlations and, as a result, of the new diagrammatic elements with the structure of Kubo cumulants and named by us as irreducible Green's functions. These functions describe the main charge, spin and pairing fluctuations of the system.

The new diagram technique for such strongly correlated systems has been developed in our earlier papers [8-18]. This diagram technique uses the algebra of Fermi operators and relies on the generalized Wick theorem which contains, apart from usual Feynman contributions, additional irreducible structures. These structures are the main elements of the diagrams.

In superconducting state, unlike the normal one, the irreducible Green's functions can contain any even number of fermion creation and annihilation operators, whereas in normal state the number of both kinds is equal. Therefore we need an automatic mathematical mechanism which 
takes into account all the possibilities to consider the interference of the particles and holes in the superconducting state.

With this purpose we use the notion of charge quantum number, introduced by us in [8] and called $\alpha$-number, which has two values $\alpha= \pm 1$ according to the definition

$$
C^{\alpha}= \begin{cases}C, & \alpha=1 \\ C^{+}, & \alpha=-1\end{cases}
$$

were $C$ is a fermion annihilation operator. In this new representation the tunneling part of the Hubbard Hamiltonian can be rewritten in the form

$$
\begin{aligned}
& H^{\prime}=\sum_{\sigma \mathbf{x x}^{\prime}} \sum t\left(\mathbf{x}^{\prime}-\mathbf{x}\right) C_{\mathbf{x}^{\prime} \sigma}^{+} C_{\mathbf{x} \sigma}= \\
& =\frac{1}{2} \sum_{\alpha=-1,1} \sum_{\sigma \mathbf{x x}^{\prime}} \alpha t_{\alpha}\left(\mathbf{x}^{\prime}-\mathbf{x}\right) C_{\mathbf{x}^{\prime} \sigma}^{-\alpha} C_{\mathbf{x} \sigma}^{\alpha},
\end{aligned}
$$

with the definition of the tunneling matrix elements

$$
\begin{aligned}
& t_{1}\left(\mathbf{x}^{\prime}-\mathbf{x}\right)=t\left(\mathbf{x}^{\prime}-\mathbf{x}\right), \\
& t_{-1}\left(\mathbf{x}^{\prime}-\mathbf{x}\right)=t\left(\mathbf{x}-\mathbf{x}^{\prime}\right), \\
& t(\mathbf{x}=0)=0 .
\end{aligned}
$$

In this charge quantum number representation the operator $H^{\prime}$ has an additional multiple $\alpha$ for every vertex of the diagrams and additional summation over $\alpha$. All the Green's functions depend of this number.

In interaction representation operator $H^{\prime}$ has a form

$$
H^{\prime}(\tau)=\frac{1}{2} \sum_{\alpha \sigma \mathbf{x x}^{\prime}} \alpha t_{\alpha}\left(\mathbf{x}^{\prime}-\mathbf{x}\right) C_{\mathbf{x}^{\prime} \sigma}^{-\alpha}\left(\tau+\alpha 0^{+}\right) C_{\mathbf{x} \sigma}^{\alpha}(\tau) .
$$

The main part of the Hubbard Hamiltonian

$$
\begin{aligned}
& H=H^{0}+H^{\prime}, \quad H^{0}=\sum_{i} H_{i}^{0}, \\
& H_{i}^{0}=-\mu \sum_{\sigma} C_{i \sigma}^{+} C_{i \sigma}+U n_{i \uparrow} n_{i \downarrow}
\end{aligned}
$$

contains the local part $H^{0}$, where $\mu$ is the chemical potential and $U$ is the Coulomb repulsion of the electrons. This interaction is considered as a main parameter of the model and is taken into account in zero approximation of our theory. The operator $H^{\prime}$ describes electron hopping between lattice sites of the crystal and is considered as a perturbation.

We shall use the grand canonical partition function in our thermodynamic perturbation theory.

The paper is organized in the following way. In Sec. 2 we define the one-particle Matsubara Green's functions in terms of $\alpha$ representation and develop the diagrammatic theory in the strong coupling limit.

In Sec. 3 we establish relation between the full thermodynamic potential and the renormalized one-particle Green's function in the presence of additional integration over auxiliary constant of interaction $\lambda$ and prove the sta- tionarity theorem both for a special functional consisting of skeleton diagrams and for a renormalized thermodynamic potential shown to be its equivalent.

\section{Diagrammatic theory}

We shall use the following definition of the Matsubara Green's functions in the interaction representation

$$
G^{\alpha \alpha^{\prime}}\left(x \mid x^{\prime}\right)=-\left\langle T C_{\mathbf{x} \sigma}^{\alpha}(\tau) C_{\mathbf{x}^{\prime} \sigma^{\prime}}^{-\alpha^{\prime}}\left(\tau^{\prime}\right) U(\beta)\right\rangle_{0}^{c}
$$

where $x$ stands for $(\mathbf{x}, \sigma, \tau)$, index $c$ of $\langle\ldots\rangle_{0}^{c}$ means the connected part of the diagrams and $\langle\ldots\rangle_{0}$ means thermal average with zero-order partition function

$$
\mathrm{e}^{-\beta H^{0}} / \operatorname{Tr} \mathrm{e}^{-\beta H^{0}} .
$$

We use the series expansion for the evolution operator $U(\beta)$ with some generalization because we introduce the auxiliary constant of interaction $\lambda$ and use $\lambda H^{\prime}$ instead $H^{\prime}$ :

$$
U_{\lambda}(\beta)=T \exp \left(-\lambda \int_{0}^{\beta} H^{\prime}(\tau) d \tau\right)
$$

with $T$ as the chronological operator. At the last stage of calculation this constant $\lambda$ will be put equal to 1 .

The correspondence between definition (6) and usual one [13] is the following:

$$
\begin{aligned}
& G_{\lambda}^{1,1}\left(x \mid x^{\prime}\right)=-\left\langle T C_{\mathbf{x} \sigma}(\tau) \bar{C}_{\mathbf{x}^{\prime} \sigma^{\prime}}\left(\tau^{\prime}\right) U_{\lambda}(\beta)\right\rangle_{0}^{c}= \\
& =G_{\sigma, \sigma^{\prime}}^{\lambda}\left(\mathbf{x}, \tau \mid \mathbf{x}^{\prime}, \tau^{\prime}\right), \\
& G_{\lambda}^{1,-1}\left(x \mid x^{\prime}\right)=-\left\langle T C_{\mathbf{x} \sigma}(\tau) C_{\mathbf{x}^{\prime} \sigma^{\prime}}\left(\tau^{\prime}\right) U_{\lambda}(\beta)\right\rangle_{0}^{c}= \\
& =F_{\sigma, \bar{\sigma}^{\prime}}^{\lambda}\left(\mathbf{x}, \tau \mid \mathbf{x}^{\prime}, \tau^{\prime}\right), \\
& G_{\lambda}^{-1,1}\left(x \mid x^{\prime}\right)=-\left\langle T \bar{C}_{\mathbf{x} \sigma}(\tau) \bar{C}_{\mathbf{x}^{\prime} \sigma^{\prime}}\left(\tau^{\prime}\right) U_{\lambda}(\beta)\right\rangle_{0}^{c}= \\
& =\bar{F} \frac{\lambda}{\bar{\sigma}, \sigma^{\prime}}\left(\mathbf{x}, \tau \mid \mathbf{x}^{\prime}, \tau^{\prime}\right), \\
& G_{\lambda}^{-1,-1}\left(x \mid x^{\prime}\right)=-G_{\lambda}^{1,1}\left(x^{\prime} \mid x\right) .
\end{aligned}
$$

As a result of application of the generalized Wick theorem we obtain for propagator (6) the diagrammatic contributions depicted on the Fig. 1.

In superconducting state, unlike the normal state, the propagator lines do not contain arrows which determine the processes of creation and annihilation of electrons because indices $\alpha$ can take two values $\alpha= \pm 1$ and every vertex of the diagram describes different possibilities.

In Fig. 1 the diagram (a) is the zero order propagator, the diagram (b) and more complicated diagrams of such kind are of chain type. They correspond to the contribution of the ordinary Wick theorem and give the Hubbard I approximation. The contributions of the diagrams (c) and (d) of Fig. 1 are 


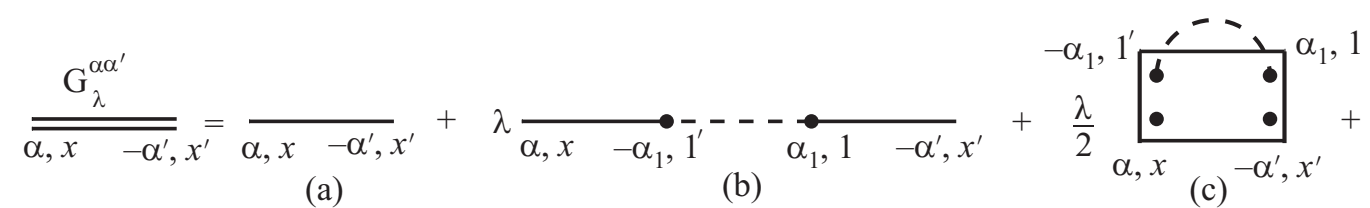

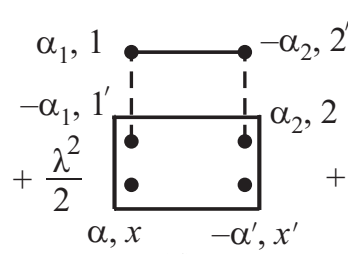

(d)

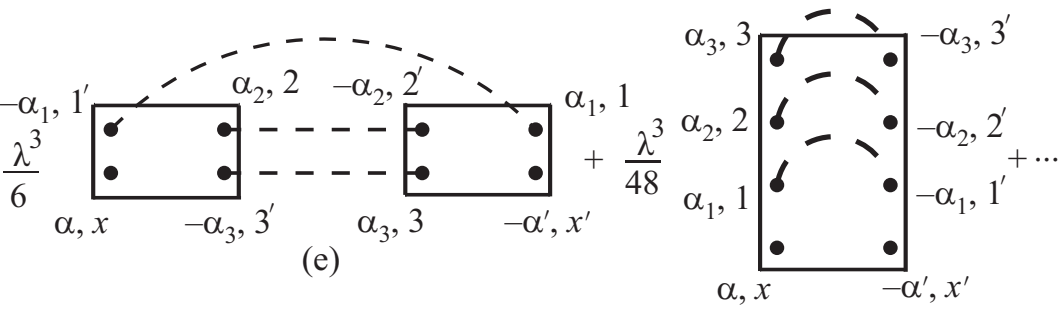

(f)

Fig. 1. The examples of the first orders perturbation theory diagrams for propagator. Solid thin lines depict zero order one-particle Green's functions and rectangles depict two- and four-particle irreducible Green's functions. Thin dashed lines correspond to tunneling matrix elements. Double solid line corresponds to renormalized propagator.

$$
\begin{aligned}
& \text { (c) : } \frac{1}{2}\left\langle T C_{\mathbf{x}}^{\alpha}(\tau) C_{\mathbf{1}^{\prime}}^{-\alpha_{1}}\left(\tau_{1}+\alpha_{1} 0^{+}\right) C_{\mathbf{1}}^{\alpha_{1}}\left(\tau_{1}\right) C_{\mathbf{x}^{\prime}}^{-\alpha^{\prime}}\left(\tau^{\prime}\right)\right\rangle_{0}^{\text {ir }} \times \\
& \times \alpha_{1} t_{\alpha_{1}}\left(\mathbf{1}^{\prime}-\mathbf{1}\right), \\
& \text { (d) }: \frac{1}{2}\left\langle T C_{\mathbf{x}}^{\alpha}(\tau) C_{\mathbf{1}^{\prime}}^{-\alpha_{1}}\left(\tau_{1}\right) C_{2}^{\alpha_{2}}\left(\tau_{2}\right) C_{\mathbf{x}^{\prime}}^{-\alpha^{\prime}}\left(\tau^{\prime}\right)\right\rangle_{0}^{\mathrm{ir}} \times \\
& \times \alpha_{1} t_{\alpha_{1}}\left(\mathbf{1}^{\prime}-\mathbf{1}\right) \alpha_{2} t_{\alpha_{2}}\left(\mathbf{2}^{\prime}-2\right) G^{(0) \alpha_{1} \alpha_{2}}\left(\mathbf{1}, \tau_{1} \mid \mathbf{2}^{\prime}, \tau_{2}\right),
\end{aligned}
$$

where $\langle\ldots\rangle_{0}^{\text {ir }}$ means the irreducible two-particle Green's function [2-5] and summation or integration is understood here and below when two repeated indices are present. Spin index has been omitted for simplicity. In the diagram (c) the equality of lattice sites indices $\mathbf{x}=\mathbf{1}^{\prime}=\mathbf{1}=\mathbf{x}^{\prime}$ is assumed and in diagram (d) $\mathbf{x}=\mathbf{1}^{\prime}=\mathbf{2}=\mathbf{x}^{\prime}$. The diagrams Fig. 1(c), (d) and (e) contain irreducible two-particles Green's functions, depicted as the rectangles. In higher orders of perturbation theory more complicated manyparticle irreducible Green's functions $G_{n}^{(0) i r}[1,2, \ldots, n]$ appear. These functions are local, i.e. with equal lattice site indices. Therefore the diagram (c) in Fig. 1 can be dropped since it contains a vanishing matrix element, $t(\mathbf{x}-\mathbf{x})=0$. The process of renormalization of the tunneling amplitude shown in the diagrams (c) and (d) leads to the replacement of the bare tunneling matrix element $\alpha t_{\alpha}\left(\mathbf{x}^{\prime}-\mathbf{x}\right)$ in (c) by a renormalized quantity $T^{\alpha \alpha^{\prime}}\left(x^{\prime} \mid x\right)$. This process is determined by the equation

$$
\begin{gathered}
T_{\sigma^{\prime} \sigma}^{\alpha^{\prime} \alpha}\left(x^{\prime} \mid x\right)=T_{\sigma^{\prime} \sigma}^{(0) \alpha^{\prime} \alpha}\left(x^{\prime} \mid x\right)+T_{\sigma^{\prime} \sigma_{1}}^{(0) \alpha^{\prime} \alpha_{1}}\left(x^{\prime} \mid x_{1}\right) \times \\
\times G_{\sigma_{1} \sigma_{2}}^{\alpha_{1} \alpha_{2}}\left(x_{1} \mid x_{2}\right) T_{\sigma_{2} \sigma}^{(0) \alpha_{2} \alpha}\left(x_{2} \mid x\right),
\end{gathered}
$$

where

$$
T_{\sigma^{\prime} \sigma}^{(0) \alpha^{\prime} \alpha}\left(x^{\prime} \mid x\right)=\delta_{\alpha \alpha^{\prime}} \alpha t_{\alpha}\left(\mathbf{x}^{\prime}-\mathbf{x}\right) \delta\left(\tau^{\prime}-\tau-\alpha 0^{+}\right) \delta_{\sigma \sigma^{\prime}},
$$

and $G^{\alpha_{1} \alpha_{2}}$ is the full one-particle propagator. The quantity $T^{\alpha^{\prime} \alpha}$ is shown in the diagrams as a double dashed line.

We then introduce the notion of correlation function $\Lambda^{\alpha \alpha^{\prime}}\left(x \mid x^{\prime}\right)$ which is the infinite sum of strongly connected parts of propagator's diagrams. If we now omit from these diagrams all those contained in the process of renormalization of the tunneling matrix element, we obtain the skeleton diagrams for correlation function. In such skeleton diagrams we replace thin dashed lines by double dashed lines and obtain the definition of $\Lambda^{\alpha \alpha^{\prime}}\left(x \mid x^{\prime}\right)$ shown in the Fig. 2.

There are two kinds of $\lambda$ dependence in the diagrams of Fig. 2. One is conditioned by dependence of $T_{\lambda}^{\alpha \alpha^{\prime}}$ and the second is determined by $\lambda$ being an explicit pre-factor in the diagrams. In Hubbard I approximation only the free propagator line is taken into account. All the contributions of Fig. 2 except the last one are local and their Fourier representation is independent of momentum. Only these diagrams are taken into account in dynamical mean field theory [19]. The last diagram of Fig. 2 has the Fourier representation which depends of momentum.

As a result of diagrammatic analysis we can formulate the Dyson-type equation for full one-particle Green's function $(x=\mathbf{x}, \tau)$ :

$$
\begin{aligned}
& G_{\sigma \sigma^{\prime}}^{\alpha \alpha^{\prime}}\left(x \mid x^{\prime}\right)=\Lambda_{\sigma \sigma^{\prime}}^{\alpha \alpha^{\prime}}\left(x \mid x^{\prime}\right)+\sum_{\sigma_{1} \sigma_{2} x_{1} x_{2}} \sum_{00}^{\beta \beta} \int_{0}^{\beta} d \tau_{1} d \tau_{2} \times \\
& \times \Lambda_{\sigma \sigma_{1}}^{\alpha \alpha_{1}}\left(x \mid x_{1}\right) T_{\sigma_{1} \sigma_{2}}^{(0) \alpha_{1} \alpha_{2}}\left(x_{1} \mid x_{2}\right) G_{\sigma_{2} \sigma^{\prime}}^{\alpha}\left(x_{2} \mid x^{\prime}\right) .
\end{aligned}
$$

This equation can be written in the operator form:

$$
\hat{G}=\left(1-\hat{\Lambda} \hat{T}^{0}\right)^{-1} \hat{\Lambda}=\hat{\Lambda}\left(1-\hat{T}^{0} \hat{\Lambda}\right)^{-1}
$$

Using Eqs. (9) and (12) we obtain the Dyson equation for the tunneling Green's function

$$
\hat{T}=\hat{T}^{0}\left(1-\hat{\Lambda} \hat{T}^{0}\right)^{-1}=\left(1-\hat{T}^{0} \hat{\Lambda}\right)^{-1} \hat{T}^{0},
$$




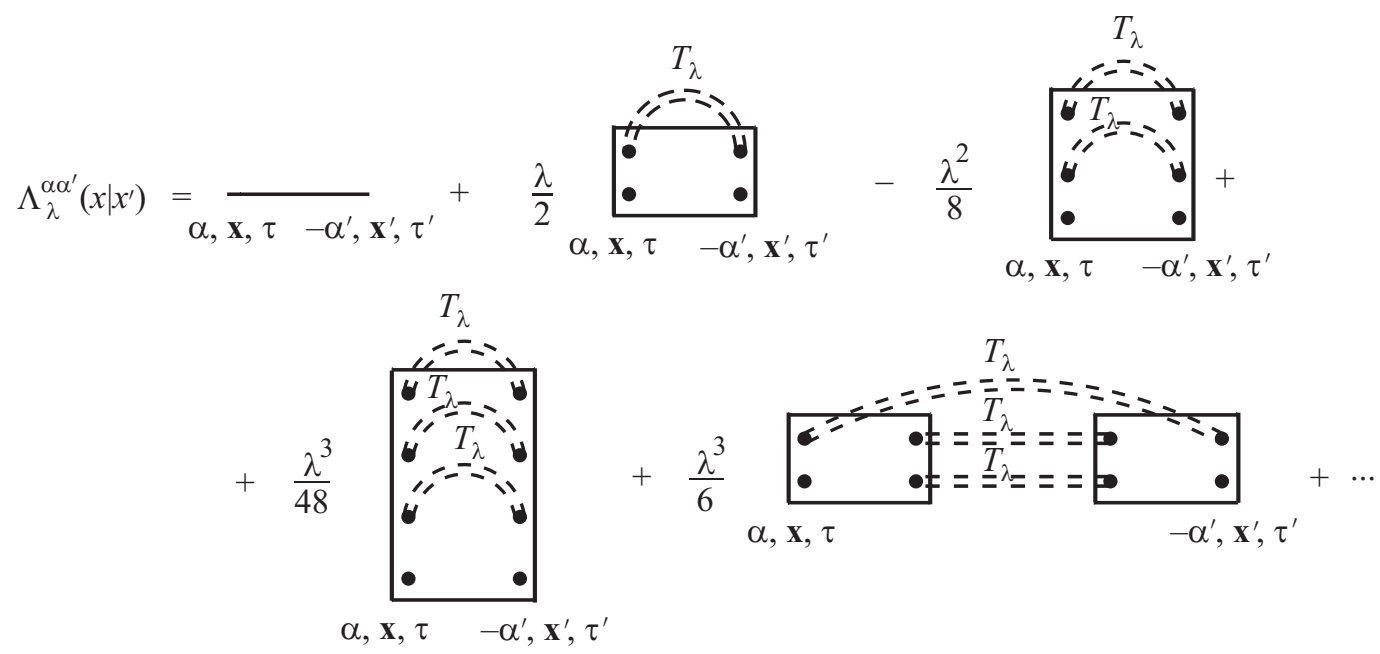

Fig. 2. The skeleton diagrams for correlation function $\Lambda^{\alpha \alpha^{\prime}}\left(x \mid x^{\prime}\right)$. The rectangles depict the many-particles irreducible Green's function. The double dashed lines depict the full tunneling Green function $T_{\lambda}^{\alpha \alpha^{\prime}}\left(x \mid x^{\prime}\right)$.

where the correlation function $\Lambda$ has the role of mass operator for the renormalized tunneling Green's function.

In Appendix A we demonstrate the equivalence of the Eq. (11) to usual [6] representation of superconducting Green's functions.

\section{Thermodynamic potential}

The thermodynamic potential of the system is determined by the connected part of the mean value of evolution operator:

$$
F(\lambda)=F_{0}-\frac{1}{\beta}\left\langle U_{\lambda}(\beta)\right\rangle_{0}^{c},
$$

with $\lambda$ equal to 1 .

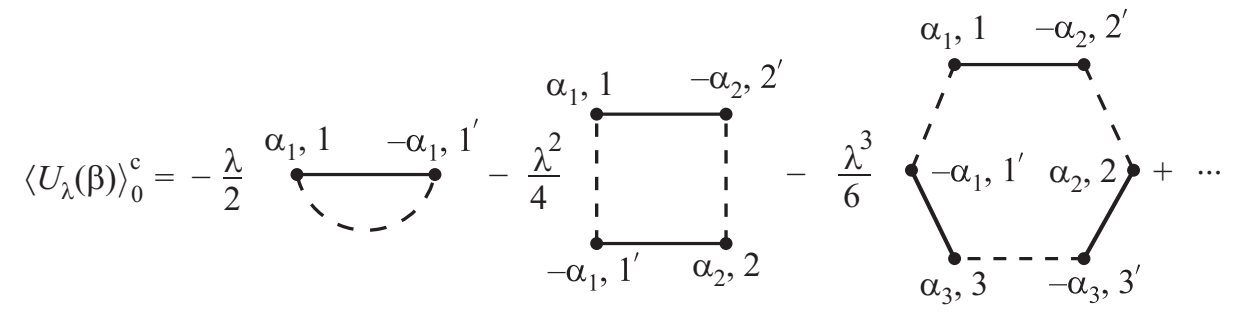

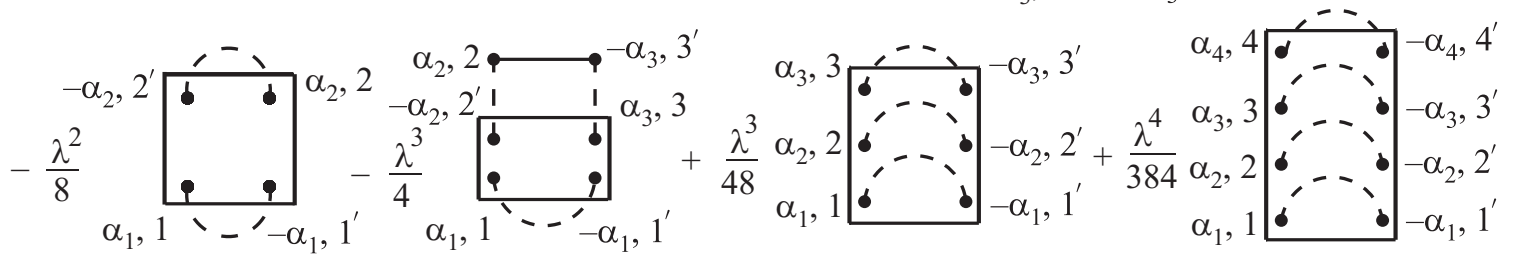

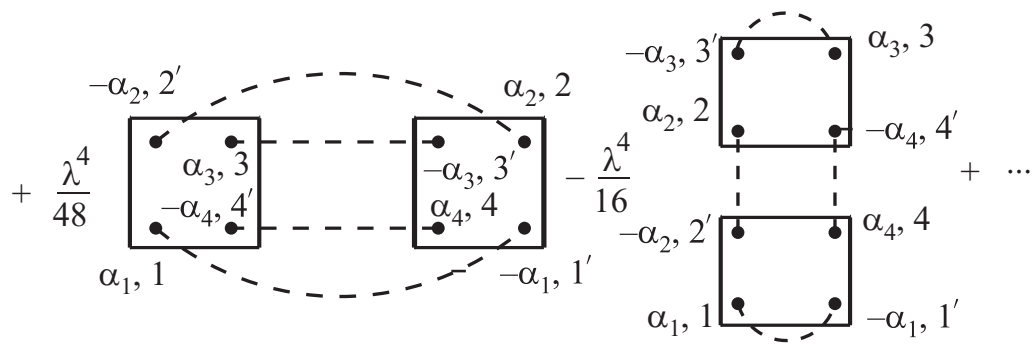

Fig. 3. The first orders of perturbation theory contributions. The skeleton diagrams for functional $Y^{\prime}(\lambda)$. 
butions of the fifth and eighth diagrams on the right-hand side of Fig. 3 are

$$
\begin{gathered}
-\frac{1}{4}\left\langle T C_{1}^{\alpha_{1}}\left(\tau_{1}\right) C_{2^{\prime}}^{-\alpha_{2}}\left(\tau_{2}\right) C_{3}^{\alpha_{3}}\left(\tau_{3}\right) C_{\mathbf{1}^{\prime}}^{-\alpha_{1}}\left(\tau_{1}+\alpha_{1} 0^{+}\right)\right\rangle_{0}^{\text {ir }} \times \\
\times \alpha_{1} t_{\alpha_{1}}\left(\mathbf{1}^{\prime}-\mathbf{1}\right) \alpha_{2} t_{\alpha_{2}}\left(\mathbf{2}^{\prime}-\mathbf{2}\right) \alpha_{3} t_{\alpha_{3}}\left(\mathbf{3}^{\prime}-3\right) \times \\
\times G^{(0) \alpha_{2} \alpha_{3}}\left(\mathbf{2}, \tau_{2} \mid \mathbf{3}^{\prime}, \tau_{3}\right), \\
+\frac{1}{48}\left\langle T C_{1}^{\alpha_{1}}\left(\tau_{1}\right) C_{2^{\prime}}^{-\alpha_{2}}\left(\tau_{2}\right) C_{3}^{\alpha_{3}}\left(\tau_{3}\right) C_{4^{\prime}}^{-\alpha_{4}}\left(\tau_{4}\right)\right\rangle_{0}^{\mathrm{ir}} \times \\
\times \alpha_{1} t_{\alpha_{1}}\left(\mathbf{1}^{\prime}-\mathbf{1}\right) \alpha_{2} t_{\alpha_{2}}\left(\mathbf{2}^{\prime}-\mathbf{2}\right) \times \\
\times\left\langle T C_{\mathbf{4}}^{\alpha_{4}}\left(\tau_{4}\right) C_{\mathbf{3}^{\prime}}^{-\alpha_{3}}\left(\tau_{3}\right) C_{2}^{\alpha_{2}}\left(\tau_{2}\right) C_{\mathbf{1}^{\prime}}^{-\alpha_{1}}\left(\tau_{1}\right)\right\rangle_{0}^{\mathrm{ir}} \times \\
\times \alpha_{3} t_{\alpha_{3}}\left(\mathbf{3}^{\prime}-\mathbf{3}\right) \alpha_{4} t_{\alpha_{4}}\left(\mathbf{4}^{\prime}-\mathbf{4}\right),
\end{gathered}
$$

respectively.

Comparison of the diagrams of Fig. 1 for the $G^{(n) \alpha \alpha^{\prime}}$ ( $n$th order of perturbation theory for the one-particle propagator) to the contributions of Fig. 2 for $\left\langle U_{\lambda}^{n+1}(\beta)\right\rangle^{c}((n+1)$-th order for evolution operator) allows us to establish the following simple relation $(n \geq 1)$ :

$$
\begin{aligned}
\left\langle U_{\lambda}^{(n+1)}(\beta)\right\rangle_{0}^{c} & =-\frac{\beta}{2} \int_{0}^{\lambda} \frac{d \lambda_{1}}{\lambda_{1}} \sum_{\mathbf{1 1}^{\prime} \alpha_{1} \sigma_{1}} \sum_{1} \lambda_{1} \alpha_{\alpha_{1}}\left(\mathbf{1}^{\prime}-\mathbf{1}\right) \times \\
& \times G_{\sigma_{1} \lambda_{1}}^{(n) \alpha_{1} \alpha_{1}}\left(\mathbf{1}-\mathbf{1}^{\prime} \mid-\alpha_{1} 0^{+}\right),
\end{aligned}
$$

and as the result we have

$$
\begin{array}{r}
\left\langle U_{\lambda}(\beta)\right\rangle_{0}^{c}=-\frac{1}{2} \int_{0}^{\lambda} \frac{d \lambda_{1}}{\lambda_{1}} \beta \sum_{\mathbf{1 1 ^ { \prime }}} \sum_{\alpha_{1} \sigma_{1}} \lambda_{1} \alpha_{1} t_{\alpha_{1}}\left(\mathbf{1}^{\prime}-\mathbf{1}\right) \times \\
\times G_{\sigma_{1} \lambda_{1}}^{\alpha_{1} \alpha_{1}}\left(\mathbf{1}-\mathbf{1}^{\prime} \mid-\alpha_{1} 0^{+}\right)=-\frac{1}{2} \int_{0}^{\lambda} \frac{d \lambda_{1}}{\lambda_{1}} \operatorname{Tr}\left(\lambda_{1} \hat{T}^{0} \hat{G}_{\lambda_{1}}\right) .
\end{array}
$$

Taking into account Eqs. (12) and (13) we obtain

$$
\left\langle U_{\lambda}(\beta)\right\rangle_{0}^{c}=-\frac{1}{2} \int_{0}^{\lambda} \frac{d \lambda_{1}}{\lambda_{1}} \operatorname{Tr}\left(\lambda_{1} \hat{T}_{\lambda_{1}} \hat{\Lambda}_{\lambda_{1}}\right) .
$$

Then from (14) and (16) it follows that

$$
\begin{aligned}
F(\lambda) & =F_{0}+\frac{1}{2 \beta} \int_{0}^{\lambda} \frac{d \lambda_{1}}{\lambda_{1}} \operatorname{Tr}\left(\lambda_{1} \hat{T}_{\lambda_{1}} \hat{\Lambda}_{\lambda_{1}}\right)= \\
& =F_{0}+\frac{1}{2 \beta} \int_{0}^{\lambda} \frac{d \lambda_{1}}{\lambda_{1}} \operatorname{Tr}\left(\hat{T}_{\lambda_{1}} \hat{\Sigma}_{\lambda_{1}}\right)
\end{aligned}
$$

where

$$
\hat{\Sigma}_{\lambda}=\lambda \hat{\Lambda}_{\lambda}
$$

has the role of mass operator for tunneling Green's function $\hat{T}_{\lambda}$. For them Dyson equation exists

$$
\hat{T}=\hat{T}^{0}+\hat{T}^{0} \hat{\Sigma} \hat{T}
$$

Equation (17) can be rewritten in the form

$$
\lambda \frac{d F(\lambda)}{d \lambda}=\frac{1}{2 \beta} \operatorname{Tr}\left(\hat{T}_{\lambda} \hat{\Sigma}_{\lambda}\right)
$$

The Eqs. (15) and (17) establish the relation between the thermodynamic potential and renormalized one-particle propagator $\hat{G}_{\lambda}$ or tunneling Green's function $\hat{T}_{\lambda}$. Both these quantities depend on auxiliary parameter $\lambda$ which is integrated over. As have been proved by Luttinger and Ward $[20,21]$, for normal state of weakly correlated systems, it is possible to obtain another expression for the thermodynamic potential without such additional integration.

In our previous paper [1], for the normal state of Hubbard model, we have obtained such an equation in the form of special functional. We now consider its generalization to the case of superconductivity. For this purpose we introduce the functional

$$
Y(\lambda)=Y_{1}(\lambda)+Y^{\prime}(\lambda)
$$

where

$$
Y_{1}(\lambda)=-\frac{1}{2} \operatorname{Tr}\left\{\ln \left(\lambda \hat{T}^{0} \hat{\Lambda}_{\lambda}-1\right)+\hat{T}_{\lambda} \lambda \hat{\Lambda}_{\lambda}\right\},
$$

and $Y^{\prime}(\lambda)$ is the functional constructed from skeleton diagrams depicted on Fig. 4.

From Figs. 2 and 4 it is possible to obtain the relation

$$
\frac{\delta Y^{\prime}(\lambda)}{\delta T_{\lambda}^{\alpha \alpha^{\prime}}\left(x \mid x^{\prime}\right)}=\frac{1}{2} \lambda \Lambda_{\lambda}^{\alpha^{\prime} \alpha}\left(x^{\prime} \mid x\right) .
$$

Now we shall take into account the following functional derivatives based on the Eqs. (12) and (13):

$$
\begin{gathered}
\frac{\delta}{\delta T_{\lambda}^{\alpha \alpha^{\prime}}\left(x \mid x^{\prime}\right)} \operatorname{Tr}\left(\ln \left(\hat{T}^{0} \hat{\Lambda} \lambda-1\right)\right)=-\operatorname{Tr}\left(\hat{T} \frac{\delta \hat{\Lambda}}{\delta T_{\lambda}^{\alpha \alpha^{\prime}}\left(x \mid x^{\prime}\right)}\right) \\
\frac{\delta}{\delta T_{\lambda}^{\alpha \alpha^{\prime}}\left(x \mid x^{\prime}\right)} \operatorname{Tr}(\hat{T} \hat{\Lambda} \lambda)=\lambda \Lambda_{\lambda}^{\alpha^{\prime} \alpha}\left(x^{\prime} \mid x\right)+ \\
+\operatorname{Tr}\left(\hat{T}_{\lambda} \frac{\delta \hat{\Lambda}_{\lambda}}{\delta T_{\lambda}^{\alpha \alpha^{\prime}}\left(x \mid x^{\prime}\right)} \lambda\right)
\end{gathered}
$$

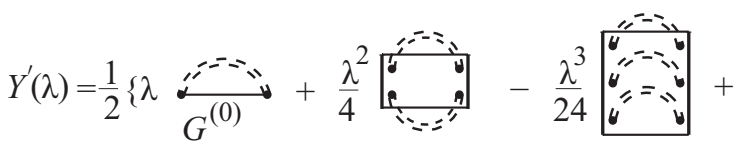

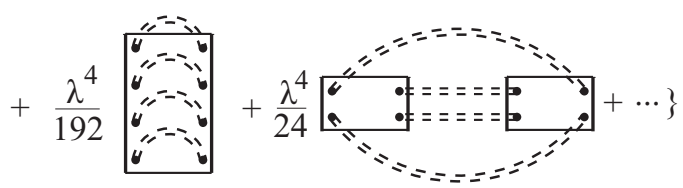

Fig. 4. The rectangles depict the irreducible Green's functions. The double dashed lines depict the tunneling renormalized Green's functions $T^{\alpha \alpha^{\prime}}\left(x \mid x^{\prime}\right)$. 
As a consequence of these equations we have

$$
\frac{\delta}{\delta T_{\lambda}^{\alpha \alpha^{\prime}}\left(x \mid x^{\prime}\right)} \operatorname{Tr}\left\{\ln \left(\lambda \hat{T}^{0} \hat{\Lambda}_{\lambda}-1\right)+\lambda \hat{T}_{\lambda} \hat{\Lambda}_{\lambda}\right\}=\lambda \Lambda_{\lambda}^{\alpha^{\prime} \alpha}\left(x^{\prime} \mid x\right),
$$

and

$$
\frac{\delta Y_{1}(\lambda)}{\delta T_{\lambda}^{\alpha \alpha^{\prime}}\left(x \mid x^{\prime}\right)}=-\frac{\lambda}{2} \Lambda_{\lambda}^{\alpha^{\prime} \alpha}\left(x^{\prime} \mid x\right)
$$

With the functional derivative of $Y^{\prime}(\lambda)$ given in (23) we obtain the stationarity property of the functional $Y(\lambda)$ :

$$
\frac{\delta Y(\lambda)}{\delta T_{\lambda}^{\alpha \alpha^{\prime}}\left(x \mid x^{\prime}\right)}=0
$$

Using the definition (18) of the mass operator $\hat{\Sigma}_{\lambda}$ we can rewrite the functional $Y_{1}(\lambda)$ in the form

$$
Y_{1}(\lambda)=-\frac{1}{2} \operatorname{Tr}\left\{\ln \left(\hat{T}^{0} \hat{\Sigma}_{\lambda}-1\right)+\hat{T}_{\lambda} \hat{\Sigma}_{\lambda}\right\}
$$

and prove the second form of stationarity property

$$
\frac{\delta Y(\lambda)}{\delta \hat{\Sigma}_{\lambda}}=0
$$

To demonstrate this equation it is sufficient to use the Dyson equation (19) in the form

$$
{\widehat{T^{0}}}^{-1}=\hat{T}_{\lambda}^{-1}+\hat{\Sigma}_{\lambda}
$$

and the derivatives:

$$
\begin{aligned}
& \frac{\delta\left(\hat{T}_{\lambda}^{-1}\right)^{\beta \beta^{\prime}}\left(y \mid y^{\prime}\right)}{\delta\left(\hat{\Sigma}_{\lambda}\right)^{\alpha \alpha^{\prime}}\left(x \mid x^{\prime}\right)}=\delta_{\alpha \beta} \delta_{\alpha^{\prime} \beta^{\prime}} \delta_{x y} \delta_{x^{\prime} y^{\prime}}, \\
& \frac{\delta\left(\hat{T}_{\lambda}\right)^{\beta \beta^{\prime}}\left(y \mid y^{\prime}\right)}{\delta\left(\hat{\Sigma}_{\lambda}\right)^{\alpha \alpha^{\prime}}\left(x \mid x^{\prime}\right)}=\left(\hat{T}_{\lambda}\right)^{\alpha^{\prime} \beta^{\prime}}\left(x^{\prime} \mid y^{\prime}\right)\left(\hat{T}_{\lambda}\right)^{\beta \alpha}(y \mid x), \\
& \frac{\delta}{\delta\left(\hat{\Sigma}_{\lambda}\right)^{\alpha \alpha^{\prime}}\left(x \mid x^{\prime}\right)} \operatorname{Tr}\left(\hat{T}_{\lambda} \hat{\Sigma}_{\lambda}\right)=\left(\hat{T}_{\lambda}\right)^{\alpha^{\prime} \alpha}\left(x^{\prime} \mid x\right)+ \\
& +\left(\hat{T}_{\lambda} \hat{\Sigma}_{\lambda} \hat{T}_{\lambda}\right)^{\alpha^{\prime} \alpha}\left(x^{\prime} \mid x\right), \\
& \frac{\delta}{\delta\left(\hat{\Sigma}_{\lambda}\right)^{\alpha \alpha^{\prime}}\left(x \mid x^{\prime}\right)} \operatorname{Tr}\left\{\ln \left(\hat{T}^{0} \hat{\Sigma}_{\lambda}-1\right)\right\}=-\left(\hat{T}_{\lambda}\right)^{\alpha^{\prime} \alpha}\left(x^{\prime} \mid x\right) \text {. }
\end{aligned}
$$

Therefore we have

$$
\frac{\delta Y_{1}(\lambda)}{\delta\left(\hat{\Sigma}_{\lambda}\right)^{\alpha \alpha^{\prime}}\left(x \mid x^{\prime}\right)}=-\frac{1}{2}\left(\hat{T}_{\lambda} \hat{\Sigma}_{\lambda} \hat{T}_{\lambda}\right)^{\alpha^{\prime} \alpha}\left(x^{\prime} \mid x\right)
$$

and

$$
\begin{gathered}
\frac{\delta Y^{\prime}(\lambda)}{\delta\left(\hat{\Sigma}_{\lambda}\right)^{\alpha \alpha^{\prime}}\left(x \mid x^{\prime}\right)}=\frac{\delta Y^{\prime}(\lambda)}{\delta\left(\hat{T}_{\lambda}\right)^{\beta \beta^{\prime}}\left(y \mid y^{\prime}\right)} \frac{\delta\left(\hat{T}_{\lambda}\right)^{\beta \beta^{\prime}}\left(y \mid y^{\prime}\right)}{\delta\left(\hat{\Sigma}_{\lambda}\right)^{\alpha \alpha^{\prime}}\left(x \mid x^{\prime}\right)}= \\
=\frac{1}{2}\left(\hat{T}_{\lambda} \hat{\Sigma}_{\lambda} \hat{T}_{\lambda}\right)^{\alpha^{\prime} \alpha}\left(x^{\prime} \mid x\right),
\end{gathered}
$$

where the usual convention about summation over the repeated indices has been adopted.

As a result we obtain the stationarity property (29) of the functional $Y(\lambda)$ versus the change of the mass operator $\Sigma_{\lambda}$. This mass operator for $\lambda=1$ coincides with correlation function of our strongly correlated model.

Now it is necessary to find a relation between the thermodynamic potential $F(\lambda)$ and the functional $Y(\lambda)$.

Consider first the value of the derivative $d Y(\lambda) / d \lambda$. The $\lambda$ dependence of the functional $Y(\lambda)$ is of two kinds: through $\Sigma_{\lambda}$ and also explicit through the factors $\lambda^{n}$ in front of the skeleton diagrams for the functional $Y^{\prime}(\lambda)$.

Due the stationarity property (29) we obtain

$$
\frac{d Y(\lambda)}{d \lambda}=\frac{\delta Y(\lambda)}{\delta \Sigma_{\lambda}} \frac{d \Sigma_{\lambda}}{d \lambda}+\left.\frac{\partial Y(\lambda)}{\partial \lambda}\right|_{\Sigma_{\lambda}}=\left.\frac{d Y(\lambda)}{d \lambda}\right|_{\Sigma_{\lambda}}=\left.\frac{d Y^{\prime}(\lambda)}{d \lambda}\right|_{\Sigma_{\lambda}} .
$$

Here we took into account that the $Y_{1}(\lambda)$ part of functional $Y(\lambda)$ (see Eqs. (21) and (28)) does not explicitly dependent on $\lambda$.

By using the definitions of $Y^{\prime}(\lambda)$ (see Fig. 4) and of $\Lambda_{\lambda}$ (see Fig. 2) it is easy to establish the property:

$$
\lambda \frac{d Y(\lambda)}{d \lambda}=\left.\lambda \frac{\partial Y^{\prime}(\lambda)}{\partial \lambda}\right|_{\Sigma_{\lambda}}=\frac{1}{2 \beta} \operatorname{Tr}\left(\lambda \hat{T}_{\lambda} \hat{\Lambda}_{\lambda}\right)=\frac{1}{2 \beta} \operatorname{Tr}\left(\hat{T}_{\lambda} \hat{\Sigma}_{\lambda}\right) .
$$

From the Eqs. (20) and (35) we have

$$
\lambda \frac{d Y(\lambda)}{d \lambda}=\frac{1}{2 \beta} \operatorname{Tr}\left(\hat{T}_{\lambda} \hat{\Sigma}_{\lambda}\right)=\lambda \frac{d F(\lambda)}{d \lambda}
$$

and we therefore obtain

$$
F(\lambda)=Y(\lambda)+F_{0},
$$

since for $\lambda=0$ the perturbation is absent $Y(\lambda=0)=0$ and $F(\lambda=0)=F_{0}$. Now we set $\lambda=1$ and obtain

$$
F=F_{0}+Y(1)
$$

with the stationarity property

$$
\frac{\delta F}{\delta \hat{\Sigma}}=0
$$

Stationary property (39) helps to obtain such thermodynamical quantities as entropy and specific heat $S=-d F / d T, \quad C=T(d S / d T)$. Thermodynamic potential depends of temperature in two forms: one dependence is explicit and second is through mass operator and because of $d F / d T=\partial F / \partial T+(\delta F / \delta \Sigma)(d \Sigma / d T)$, as a conse- 
quence of (39) the second term in the last formula can be omitted.

\section{Conclusions}

We have further developed the diagrammatic theory proposed for strongly correlated systems many years ago to establish the stationarity property of the thermodynamic potential in the superconducting state of the Hubbard model.

First, we have introduced the notion of charge quantum number which gives the possibility to consider the presence of irreducible Green's functions with an arbitrary number of creation or annihilation Fermi operators in superconducting state.

We have introduced the notion of tunneling Green's function and its mass operator, which turns out to be equal to the correlation function of the fermion system.

We have proven the existence of the Dyson equation for this function and establish the exact relation between the thermodynamic potential and renormalized one-particle propagator. This relation contains an additional integration over the auxiliary constant of interaction $\lambda$.

We have constructed a special functional based on the skeleton diagrams for the propagator and for the evolution operator which contain the irreducible Green's functions and full tunneling Green's functions.

We have proven the existence of the stationarity property of this functional and establish its relation with thermodynamic potential.

It is important to emphasize that there is a close similarity between our results obtained for two different models of strongly correlated systems such as periodic Anderson model (PAM) and the Hubbard model (HM). From comparison of the results obtained for the PAM (see paper [18]) and the results of the present paper for the HM the topological coincidence of the diagrams for both models has been revealed.

For example the skeleton diagrams of Fig. 3 of paper [18], obtained for $\Lambda$ functional of PAM topologically coincide with the skeleton diagrams of our Fig. 2 for the same functional, but of quite a different model. In order to obtain a complete coincidence, it is necessary to replace the full Green's function $G_{C}(i \omega)$ of conduction electrons of PAM by the renormalized tunneling Green's function $T(i \omega)$ of the HM.

The same similarity exists between other functionals of these models. For example, comparison of the skeleton diagrams of Fig. 10 of paper [18] with the diagrams of Fig. 4 of the present paper reveals the full coincidence upon replacement of the Green's functions $G_{C}$ by $T$. This comparison allows us to conclude that from the thermodynamic point of view the PAM can be reduced to the HM if we replace the Green's function of the conduction electrons of PAM subsystem by tunneling Green's function of hopping electrons of HM.

We also note that the skeleton representation of our functional allows to select the local irreducible Green's functions as can be seen from Fig. 2 of our paper and Fig. 10 of paper [18]. These quantities contain only fluctuations in time, unlike the nonlocal ones which include both fluctuations in time and space. The coefficients of local diagrams (see Fig. 10) vary with the order of perturbation theory as $1 /\left(2^{n-1} n\right.$ !) for $n>1$.

Only such local diagrams are relevant for DMFT, so that one can attempt to carry out the summation of this class of diagrams.

Two of us (V.A.M and L.A.D) would like to thank Professor N.M. Plakida and Dr. S. Cojocaru for a very helpful discussion.

Appendix A.

\section{Gor'kov-Nambu representation}

We consider Eq. (11) in Fourier representation. By inserting specific values of charge quantum number $\lambda= \pm 1$ we obtain $\left(k=\left(\mathbf{k}, i \omega_{n}\right)\right)$

$$
\begin{gathered}
G_{\sigma \sigma^{\prime}}^{1,1}(k)=\Lambda_{\sigma \sigma^{\prime}}^{1,1}(k)+\Lambda_{\sigma \sigma_{1}}^{1,1}(k) \epsilon_{1}(\mathbf{k}) G_{\sigma_{1} \sigma^{\prime}}^{1,1}(k)-\Lambda_{\sigma,-\sigma_{1}}^{1,-1}(k) \epsilon_{-1}(\mathbf{k}) G_{-\sigma_{1}, \sigma^{\prime}}^{-1,1}(k), \\
G_{\sigma,-\sigma^{\prime}}^{1,-1}(k)=\Lambda_{\sigma,-\sigma^{\prime}}^{1,-1}(k)+\Lambda_{\sigma, \sigma_{1}}^{1,1}(k) \epsilon_{1}(\mathbf{k}) G_{\sigma_{1},-\sigma^{\prime}}^{1,-1}(k)+\Lambda_{\sigma,-\sigma_{1}}^{1,-1}(k) \epsilon_{-1}(\mathbf{k}) G_{-\sigma^{\prime},-\sigma}^{1,1}(-k), \\
G_{-\sigma, \sigma^{\prime}}^{-1,1}(k)=\Lambda_{-\sigma, \sigma^{\prime}}^{-1,1}(k)+\Lambda_{-\sigma, \sigma_{1}}^{-1,1}(k) \epsilon_{1}(\mathbf{k}) G_{\sigma_{1}, \sigma^{\prime}}^{1,1}(k)+\Lambda_{-\sigma_{1},-\sigma}^{1,1}(-k) \epsilon_{-1}(\mathbf{k}) G_{-\sigma_{1}, \sigma^{\prime}}^{-1,1}(k), \\
G_{-\sigma^{\prime},-\sigma}^{1,1}(-k)=\Lambda_{-\sigma_{,}^{\prime},-\sigma}^{1,1}(-k)-\Lambda_{-\sigma, \sigma_{1}}^{-1,1}(k) \epsilon_{1}(\mathbf{k}) G_{\sigma_{1},-\sigma^{\prime}}^{1,-1}(k)+\Lambda_{-\sigma_{1},-\sigma}^{1,1}(-k) \epsilon_{-1}(\mathbf{k}) G_{-\sigma^{\prime},-\sigma_{1}}^{1,1}(-k) .
\end{gathered}
$$

Here

$$
\epsilon_{1}(\mathbf{k})=\epsilon(\mathbf{k}), \epsilon_{-1}(\mathbf{k})=\epsilon(-\mathbf{k}), \epsilon(\mathbf{k})=\frac{1}{N} \sum_{\mathbf{x}} t(\mathbf{x}) \mathrm{e}^{i \mathbf{k} x}, \quad \sum_{\mathbf{k}} \epsilon(\mathbf{k})=0, \quad G_{\sigma \sigma^{\prime}}^{-1,-1}(k)=-G_{\sigma^{\prime} \sigma}^{1,1}(-k)
$$


Assuming that the system is in a paramagnetic state, that superconductivity has a singlet character and using the definitions (8) together with the additional ones:

$$
\begin{aligned}
& \Lambda_{\sigma \sigma}^{1,1}(k)=\Lambda_{\sigma}(k), \quad \Lambda_{\sigma \bar{\sigma}}^{1,-1}(k)=Y_{\sigma \bar{\sigma}}(k), \\
& \Lambda_{\bar{\sigma} \sigma}^{-1,1}(k)=\bar{Y}_{\bar{\sigma} \sigma}(k),
\end{aligned}
$$

we obtain the following results:

$$
\begin{gathered}
G_{\sigma}(k)=\frac{\Lambda_{\sigma}(k)\left(1-\epsilon(-\mathbf{k}) \Lambda_{\bar{\sigma}}(-k)\right)-\epsilon(-\mathbf{k}) Y_{\sigma \bar{\sigma}}(k) \bar{Y}_{\bar{\sigma} \sigma}(k)}{d_{\sigma}(k)} \\
F_{\sigma \bar{\sigma}}(k)=\frac{Y_{\sigma \bar{\sigma}}(k)}{d_{\sigma}(k)}, \bar{F}_{\bar{\sigma} \sigma}(k)=\frac{\bar{Y}_{\bar{\sigma} \sigma}(k)}{d_{\sigma}(k)}, \\
d_{\sigma}(k)=\left(1-\epsilon(\mathbf{k}) \Lambda_{\sigma}(k)\right)\left(1-\epsilon(-\mathbf{k}) \Lambda_{\bar{\sigma}}(-k)\right)+ \\
+\epsilon(\mathbf{k}) \epsilon(-\mathbf{k}) Y_{\sigma \bar{\sigma}}(k) \bar{Y}_{\bar{\sigma} \sigma}(k)
\end{gathered}
$$

which coincide with those found in the papers [10,11].

In spinor representation the system of Eqs. (A.1)-(A.7) has the form

$$
\hat{G}=\hat{\Lambda}+\hat{\Lambda} \hat{\epsilon} \hat{G}
$$

were

$$
\begin{gathered}
\hat{G}=\left(\begin{array}{cc}
G_{\sigma}(k) & F_{\sigma \bar{\sigma}}(k) \\
\bar{F}_{\bar{\sigma} \sigma}(k) & -G_{\bar{\sigma}}(-k)
\end{array}\right), \\
\hat{\Lambda}=\left(\begin{array}{cc}
\Lambda_{\sigma}(k) & Y_{\sigma \bar{\sigma}}(k) \\
\bar{Y}_{\bar{\sigma} \sigma}(k) & -\Lambda_{\bar{\sigma}}(-k)
\end{array}\right), \quad \hat{\epsilon}=\left(\begin{array}{cc}
\epsilon(k) & 0 \\
0 & -\epsilon(-k)
\end{array}\right) .
\end{gathered}
$$

By using Eq. (9) we can obtain

$$
\begin{aligned}
& T_{\sigma}^{1,1}(k)=\frac{\epsilon(\mathbf{k})\left(1-\epsilon(-\mathbf{k}) \Lambda_{\bar{\sigma}}(-k)\right)}{d_{\sigma}(k)}, \\
& T_{\sigma \bar{\sigma}}^{1,-1}(k)=-\epsilon(\mathbf{k}) \epsilon(-\mathbf{k}) F_{\sigma \bar{\sigma}}(k), \\
& T_{\bar{\sigma} \sigma}^{-1,1}(k)=-\epsilon(\mathbf{k}) \epsilon(-\mathbf{k}) \bar{F}_{\bar{\sigma} \sigma}(k) .
\end{aligned}
$$

1. V.A. Moskalenko, L.A. Dohotaru, and I.D. Cebotari, Zh. Eksp. Teor. Fiz. 138, 107 (2010).

2. J. Hubbard, Proc. Roy. Soc. A276, 238 (1963).

3. J. Hubbard, Proc. Roy. Soc. A281, 401 (1964).

4. J. Hubbard, Proc. Roy. Soc. A285, 542 (1965).

5. N.N. Bogolyubov, Full Collection of Papers, Vol. 10, Nauka, Moscow (2007).

6. A.A. Abrikosov, L.P. Gor'kov, and I.E. Dzyaloshinsky, The Method of Quantum Field Theory in Statistical Physics, Dobrosvet, Moscow (1998).

7. M. Sigrist, Introduction to Unconventional Superconductivity, ETH Zurich, Lectures WS05/06 (2006), p.79.

8. M.I. Vladimir and V.A. Moskalenko, Teor. Mat. Fiz. 82, 428 (1990) [Theor. Math. Phys. 82, 301 (1990)].

9. S.I. Vakaru, M.I. Vladimir, and V.A. Moskalenko, Teor. Mat. Fiz. 85, 248 (1990) [Theor. Math. Phys. 85, 185 (1990)].

10. N.N. Bogoliubov and V.A. Moskalenko, Teor. Mat. Fiz. 86, 16 (1991) [Theor. Math. Phys. 86, 10 (1991)]; Doklady AN SSSR 316, 1107 (1991); JINR Rapid Communications 44, 5 (1990).

11. N.N. Bogoliubov and V.A. Moskalenko, Teor. Mat. Fiz. 92, 182 (1992) [Theor. Math. Phys. 92, 820 (1992)].

12. V.A. Moskalenko, Teor. Mat. Fiz. 110, 308 (1997) [Theor. Math. Phys. 110, 243 (1997)].

13. V.A. Moskalenko, Teor. Mat. Fiz. 116, 456 (1998) [Theor. Math. Phys. 116, 1094 (1998)].

14. V.A. Moskalenko, P. Entel, and D.F. Digor, Phys. Rev. B 59, 619 (1999).

15. V.A. Moskalenko, P. Entel, D.F. Digor, L.A. Dohotaru, and R. Citro, Teor. Mat. Fiz. 155, 914 (2008) [Theor. Math. Phys. 155, 535 (2008)].

16. V.A. Moskalenko, P. Entel, L.A. Dohotaru, D.F. Digor, and R. Citro, Diagrammatic Theory for Anderson Impurity Model, Preprint BLTP, JINR, Dubna, E17-2008-56.

17. V.A. Moskalenko, P. Entel, L.A. Dohotaru, and R. Citro, Teor. Mat. Fiz. 159, 500 (2009) [Theor. Math. Phys 159, 454 (2009)].

18. V.A. Moskalenko, L.A. Dohotaru, and R. Citro, Teor. Mat. Fiz. 162, 439 (2010) [Theor. Math. Phys. 162, 366 (2010)].

19. A. Georges, G. Kotliar, W. Krauth, and M.J. Rozenberg, Rev. Mod. Phys. 8, 13 (1996); G. Kotliar and D. Vollhardt, Physics Today 57, 53 (2004).

20. J.M. Luttinger and J.C. Ward, Phys. Rev. 118, 1417 (1960).

21. J.M. Luttinger, Phys. Rev. 119, 1153 (1960). 